\title{
Fisher information and Rényi entropies in dynamical systems
}

\author{
B. Godó and Á. Nagy \\ Department of Theoretical Physics, University of Debrecen,H-4010 Debrecen, Hungary
}

(Dated: June 2, 2017)

\begin{abstract}
The link between the Fisher information and Rényi entropies is explored. The relationship is based on a thermodynamical formalism based on Fisher information with a parameter, $\beta$, which is interpreted as the inverse temperature. The Fisher heat capacity is defined and found to be sensitive to changes of higher order than the analogous quantity in the conventional formulation.
\end{abstract}


Chaotic dynamics are mathematically analogous to statistical mechanics. This similarity to thermodynamics has proved to be very useful in several applications. Now it is demonstrated that it is possible to construct a thermodynamic picture based on Fisher information. This new picture has a Legendre structure with 'Fisher' thermodynamic potentials. The present formalism offers a new insight into chaotic dynamics, resulting, for example, in a link between the Fisher information and Rényi entropies and providing a sensitive description of fluctuations utilising Fisher heat capacity.

\section{INTRODUCTION}

Fisher information [1] has received an enormous interest in several fields in physics [2]. Remarkably, a Legendre transform structure reflecting classical thermodynamics has been presented $[2,3]$. Even Fisher temperature was defined and Fisher thermodynamics was introduced $[2,4,5]$. Recently another Fisher thermodynamics has been formulated [6]. It is based on Fisher information with a parameter that is taken the inverse temperature of canonical thermodynamics. It is equivalent to traditional thermodynamics and possesses the proper Legendre structure.

In our previous paper [7] this new formalism was generalized to chaotic systems. The thermodynamics of chaotic systems [8-12] is a useful technique for studying multifractals. It is based on a resemblance between chaos theory and statistical mechanics. We demonstrated that a similar analogy can be found using a Fisher information based thermodynamic scheme. That paper focused on the thermostatistics of chaotic systems. Here, the formalism concentrates on the dynamics of chaos. This novel interpretation yields further enlightenment into chaos theory: a link between the Fisher information and Rényi entropies is explored and a quantity - Fisher heat capacity - sensitive to high order fluctuations is defined.

The layout of this paper is as follows: the following section outlines the alternative formulation of the thermodynamics of Porporato [6]. The Rényi entropies and the spectra

of dynamical scaling indices are summarized in Sections III. Section IV presents the Fisher information based thermodynamic description of dynamical systems. Section V is devoted to an illustrative example and discussion. 


\section{THERMODYNAMICS WITH FISHER INFORMATION}

First, the thermodynamic formalism based on Fisher information is summarized [6]. In a canonical ensemble the macrostate of a system is specified by the volume $V$, the particle number $N$ and the temperature $T$. The canonical distribution of a random variable associated with the energy $\hat{U}$, is given by

$$
p(\hat{U} ; \beta)=\frac{g(\hat{U})}{Z(\beta)} e^{-\beta \hat{U}}=g(\hat{U}) e^{-\Phi(\beta)-\beta \hat{U}},
$$

where $\beta=1 / T$ is the inverse temperature and $g(\hat{U})$ is the density of states. The partition function $Z(\beta)$ can be written as

$$
Z(\beta)=\int g(\hat{U}) e^{-\beta \hat{U}} d \hat{U}
$$

and

$$
Z(\beta)=\sum_{r} g_{r} e^{-\beta \hat{U}_{r}}
$$

for continuous and discrete probability distributions, respectively. (Units where the Boltzmann constant is equal to 1 are used.) The Massieu function $\Phi(\beta)$ can be expressed by the partition function $Z(\beta)$ as

$$
\Phi(\beta)=\ln (Z(\beta))=-\frac{F}{T}
$$

where $F$ is the Helmholtz free energy.

The first derivative of Massieu function $\Phi(\beta)$ with respect to the inverse temperature $\beta$ is the average of the energy

$$
-\frac{\partial \Phi(\beta)}{\partial \beta}=\langle\hat{U}\rangle=U
$$

while the second derivative is the variance of the energy $\hat{U}$ :

$$
\mathcal{F}_{U}=\frac{\partial^{2} \Phi(\beta)}{\partial \beta^{2}}=(U-\langle\hat{U}\rangle)^{2} .
$$

The variance of the energy $\hat{U}$ is equal to the Fisher information:

$$
\mathcal{F}_{U}=\frac{\partial^{2} \Phi(\beta)}{\partial \beta^{2}}=I
$$


that is usually written in the form

$$
I(\beta)=\int p(x \mid \beta)\left[\frac{\partial \ln p(x \mid \beta)}{\partial \beta}\right]^{2} d x=\int \frac{1}{p(x \mid \beta)}\left[\frac{\partial p(x \mid \beta)}{\partial \beta}\right]^{2} d x
$$

and

$$
I(\beta)=\sum_{i} p_{i}(\beta)\left[\frac{\partial \ln p_{i}(\beta)}{\partial \beta}\right]^{2}=\sum_{i} \frac{1}{p_{i}(\beta)}\left[\frac{\partial p_{i}(\beta)}{\partial \beta}\right]^{2}
$$

for continuous and discrete probability distributions, respectively. The Fisher information can also be expressed in terms of the heat capacity $C$ as

$$
\mathcal{F}_{U}=\frac{C(\beta)}{\beta^{2}}=C(T) T^{2} .
$$

In the entropy representation the Legendre transformation of the entropy $S(U, V, N)$ leads to the Massieu function

$$
\Phi=S-\beta U=-\frac{F}{T}
$$

While the entropy $S(U, V, N)$ is a fundamental function of the variables $U, V$ and $N$ with

$$
\frac{\partial S}{\partial U}=\beta
$$

the Massieu function $\Phi(T, V, N)$ is a fundamental function of the variables $T, V$ and $N$ with the fundamental relation

$$
\frac{\partial \Phi}{\partial \beta}=-U
$$

(In the following the dependence on the fixed quantities $V$ and $N$ is suppressed.)

Defining the function $\mathcal{F}_{\mathcal{S}}$ by

$$
\mathcal{F}_{S}=U+\beta \mathcal{F}_{U}
$$

one can see the correspondence between the functions $S(U)$ and $\mathcal{F}_{S}\left(\mathcal{F}_{U}\right)$. The Legendre transformation (11) resembles to $U=\mathcal{F}_{S}-\beta \mathcal{F}_{U}$ which is equivalent to Eq. (14). The fundamental relation

$$
\frac{\partial \mathcal{F}_{S}}{\partial \mathcal{F}_{U}}=\beta
$$

harmonizes with Eq. (12). The Legendre transform of $\mathcal{F}_{\mathcal{U}}$ can be constructed in energy representation

$$
\mathcal{F}_{F}=\mathcal{F}_{U}-T \mathcal{F}_{S}
$$


that corresponds to the usual definition of the free energy $F=U-T S$, while the relation

$$
\frac{\partial \mathcal{F}_{F}}{\partial T}=-\mathcal{F}_{S}
$$

corresponds to the expression $\partial F / \partial T=-S$. Table 1. shows the analoque quantities.

The 'Fisher' heat capacity can be defined as the derivative of the Fisher information $\mathcal{F}_{U}$

$$
\frac{\partial \mathcal{F}_{U}}{\partial T}=\mathcal{C}_{F}
$$

analogously to the heat capacity of the traditional thermodynamics.

\section{RÉNYI ENTROPIES AND THE SPECTRA OF DYNAMICAL SCALING IN- DICES}

In order to generalize the Fisher information formalism to chaotic dynamics, we first summarize the most important definitions and relations [8]. Assume that there exists a generating partition of the phase space. It incorporates a finite number of cells of variable size. A trajectory $x_{0}, x_{1}, \ldots$ generates a symbol sequence $i_{0}, i_{1}, i_{2} \ldots$. Consider a finite symbol sequence $i_{0}, \ldots, i_{N-1}$ and let $J\left[i_{0}, \ldots, i_{N-1}\right]$ indicate the set of all initial values $x_{0}$ that generate

this sequence. This set $J_{j}^{(N)} \equiv J\left[i_{0}, \ldots, i_{N-1}\right]$ is called $N$-cylinder. The number of allowed sequences is denoted by $\omega(N)$. Let

$$
p_{j}^{(N)}=p\left(i_{0}, \ldots, i_{N-1}\right), \quad j=1,2, \ldots, \omega(N)
$$

stand for the probability of the $j$ th symbol sequence. The dynamical partition function is defined as

$$
Z_{N}^{d y n}(\beta)=\sum_{j=1}^{\omega}\left(p_{j}^{(N)}\right)^{\beta}
$$

where $\beta$ is a real parameter. The Massieu function $\Phi(\beta)$ can be expressed by the dynamical partition function $Z(\beta)$ as

$$
\Phi(\beta)=\ln \left(Z_{N}^{d y n}(\beta)\right)=-\frac{F}{T},
$$

where $F$ is the Helmholtz free energy. To construct the Rényi entropy we first take the limit [13]

$$
\lim _{N \rightarrow \infty} \frac{1}{N} \frac{1}{1-\beta} \ln \left(Z_{N}^{d y n}(\beta)\right)
$$


and then take a supremum over all partitions. If we have a generating partition, then the supremum over partitions is already reached and the Rényi entropy has the form

$$
K(\beta)=\lim _{N \rightarrow \infty} \frac{1}{N} \frac{1}{1-\beta} \ln \sum_{j=1}^{\omega}\left(p_{j}^{(N)}\right)^{\beta},
$$

where

$$
p_{j}^{(N)}=\mu\left(J_{j}^{(N)}\right)=\int_{J_{j}^{(N)}} d \mu(x)
$$

with the natural invariant measure $\mu$.

The sum in Eq. (20) can be replaced by an integral, therefore

$$
\Phi(\beta)=\ln \int_{\gamma_{\min }}^{\gamma_{\max }} d \gamma W(\gamma) e^{-\beta \gamma N}
$$

where $W(\gamma) d \gamma$ is the number of symbol sequences $i_{0}, \ldots, i_{N-1}$ as the dynamical crowding index $\gamma$ varies between $\gamma$ and $\gamma+d \gamma$. For large $N$ the asymptotic scaling behaviour is

$$
W(\gamma) \sim e^{N g(\gamma)}
$$

Hence Eq. (25) takes the form

$$
\Phi \sim \ln \int_{\gamma_{\min }}^{\gamma_{\max }} d \gamma e^{[g(\gamma)-\beta \gamma] N}
$$

For large $N$

$$
\Phi \sim[g(\gamma)-\beta \gamma] N
$$

as the integrand takes its maximum and

$$
\frac{\partial g(\gamma)}{\partial \gamma}=\beta
$$

Eq. (28) can be rewritten as

$$
\Phi \sim(g-\beta \gamma) N
$$

The Legendre transformation in (11) leads to

$$
\Phi=S-\beta \gamma N
$$

In the thermodynamic limit, $g(\gamma)$ corresponds to the specific entropy $S / N$ :

$$
\lim _{N \rightarrow \infty} \frac{S}{N}=g(\gamma)
$$


The Legendre transformation of $g(\gamma)$ defines the function $\tau_{d y n}(\beta)$

$$
\tau_{d y n}(\beta)=\beta \gamma-g(\gamma)
$$

with

$$
\frac{\partial \tau_{d y n}}{\partial \beta}=\gamma
$$

Eqs. (31) -(34) lead to

$$
\lim _{N \rightarrow \infty} \frac{\Phi}{N}=-\tau_{d y n}(\beta)
$$

in the thermodynamic limit. The function $\tau_{d y n}(\beta)$ can be also expressed with the Rényi entropy $K(\beta)$ as

$$
(\beta-1) K(\beta)=\tau_{\text {dyn }}(\beta)
$$

\section{FISHER THERMODYNAMICS OF CHAOTIC DYNAMICS}

Being aware of the formalism of the traditional chaotic dynamics summarized in the

previous section, now we are ready to extend the Fisher information theory to dynamical systems. Taking into account that

$$
\tau_{d y n} \sim-\Phi
$$

and

$$
\gamma \sim U
$$

due to Eq. (11) and Eqs. (31)- (33), and (5) and (34), respectively, the Fisher information is defined as

$$
\mathcal{F}_{\gamma}=-\frac{d^{2} \tau_{d y n}(\beta)}{d \beta^{2}}=-\frac{\partial \gamma}{\partial \beta}
$$

The Legendre transformation giving rise to $\mathcal{F}_{\mathcal{S}}$ in Eq. (14), here generates

$$
\mathcal{F}_{g}=\gamma-\beta \frac{d \gamma}{d \beta}=\gamma+\beta \mathcal{F}_{\gamma}
$$

fulfilling the fundamental relation

$$
\frac{\partial \mathcal{F}_{g}}{\partial \mathcal{F}_{\gamma}}=\beta .
$$


The quantity resembling to the free energy of the usual thermodynamic formalism is

$$
\mathcal{F}_{\phi}=\mathcal{F}_{\gamma}-T \mathcal{F}_{g}
$$

Table I shows thermodynamic variables and their analogues in multifractals in the usual thermodynamic and the Fisher information representation.

The Fisher information can be related to the Rényi entropy $K(\beta)$ (Eq. (36))

$$
\mathcal{F}_{\gamma}=-\frac{d^{2} \tau_{d y n}(\beta)}{d \beta^{2}}=(1-\beta) \frac{d^{2} K}{d \beta^{2}}-2 \frac{d K}{d \beta} .
$$

Analogously to the fact that the Fisher information is the variance of the energy $U$ (Eq. (6)), here the Fisher information $\mathcal{F}_{\gamma}$ stands for the variance of $\gamma$ :

$$
\mathcal{F}_{\gamma}=(\gamma-\langle\gamma\rangle)^{2}
$$

Construct the counterpart of the heat capacity given in Eq. (10) as

$$
\mathcal{F}_{\gamma}=\frac{C_{d y n}(\beta)}{\beta^{2}}=C_{d y n}(T) T^{2}
$$

Owing to the correspondence of the Fisher information $\mathcal{F}_{\gamma}$ and $\gamma($ Eq. (5)) and $U$ another kind of heat capacity, the Fisher heat capacity is defined as the derivative of the Fisher information $\mathcal{F}_{\gamma}$ with respect to $T$ :

$$
\frac{\partial \mathcal{F}_{\gamma}}{\partial T}=\mathcal{C}_{F}^{d y n}
$$

\section{ILLUSTRATIVE EXAMPLE AND DISCUSSION}

To illustrate our Fisher formalism we select the well-known example: the asymmetric triangular map

$$
f(x)= \begin{cases}\frac{x}{w}, & \text { for } 0 \leq x \leq w \\ \frac{1-x}{1-w}, & \text { for } w \leq x \leq 1\end{cases}
$$

with $w \in(0,1)$. A partition with the two intervals $A_{0}=[0, w), A_{1}=[w, 1)$ gives the generating partition [8]. Let the symbols $L$ and $R$ stand for $A_{0} A_{1}$, respectively. An $N$ cylinder associated with a symbol sequence of length $N$ takes the width

$$
l_{j}^{(N)}=w^{M}(1-w)^{N-M},
$$


where the number of symbols $L$ in a sequence is $M$. The probability $p_{j}^{(N)}=l_{j}^{(N)}$ as the Lebesque measure is ascribed to each cylinder. The dynamical partition function (Eq. (20)) can be given by

$$
Z_{N}^{d y n}(\beta)=\sum_{j}\left(p_{j}^{(N)}\right)^{\beta}=\left[w^{\beta}+(1-w)^{\beta}\right]^{N}
$$

Therefore, the Rényi entropy (Eq. (23)) takes the form

$$
K(\beta)=\frac{1}{1-\beta} \ln \left[w^{\beta}+(1-w)^{\beta}\right]
$$

and

$$
\tau_{d y n}(\beta)=(\beta-1) K(\beta)=-\ln \left[w^{\beta}+(1-w)^{\beta}\right]
$$

Fig. 1 presents $\tau_{d y n}(\beta)$ and $K(\beta)$ for $w=0.3$. If $w<1-w$ (as in our case)

$$
\lim _{\beta \rightarrow-\infty} K(\beta)=-\ln w
$$

and

$$
\lim _{\beta \rightarrow+\infty} K(\beta)=-\ln (1-w)
$$

These limits are important as they describe the scaling behaviour of the largest $(\beta \rightarrow+\infty)$ and the smallest $(\beta \rightarrow-\infty)$ symbol sequence probability. For large absolute values of $\beta$ we are led to

$$
\begin{gathered}
K(\beta) \sim \frac{\beta}{1-\beta} \gamma_{\min }=\frac{\beta}{1-\beta} \ln w \quad \text { for } \quad \beta \gg 1 \\
K(\beta) \sim \frac{\beta}{1-\beta} \gamma_{\max }=\frac{\beta}{1-\beta} \ln (1-w) \quad \text { for } \quad \beta \ll 1 .
\end{gathered}
$$

On the other hand,

$$
\tau_{d y n}(\beta) \sim-\beta \ln w \quad \text { for } \quad \beta \ll 1 \text {. }
$$

and

$$
\tau_{d y n}(\beta) \sim-\beta \ln (1-w) \quad \text { for } \quad \beta \gg 1
$$




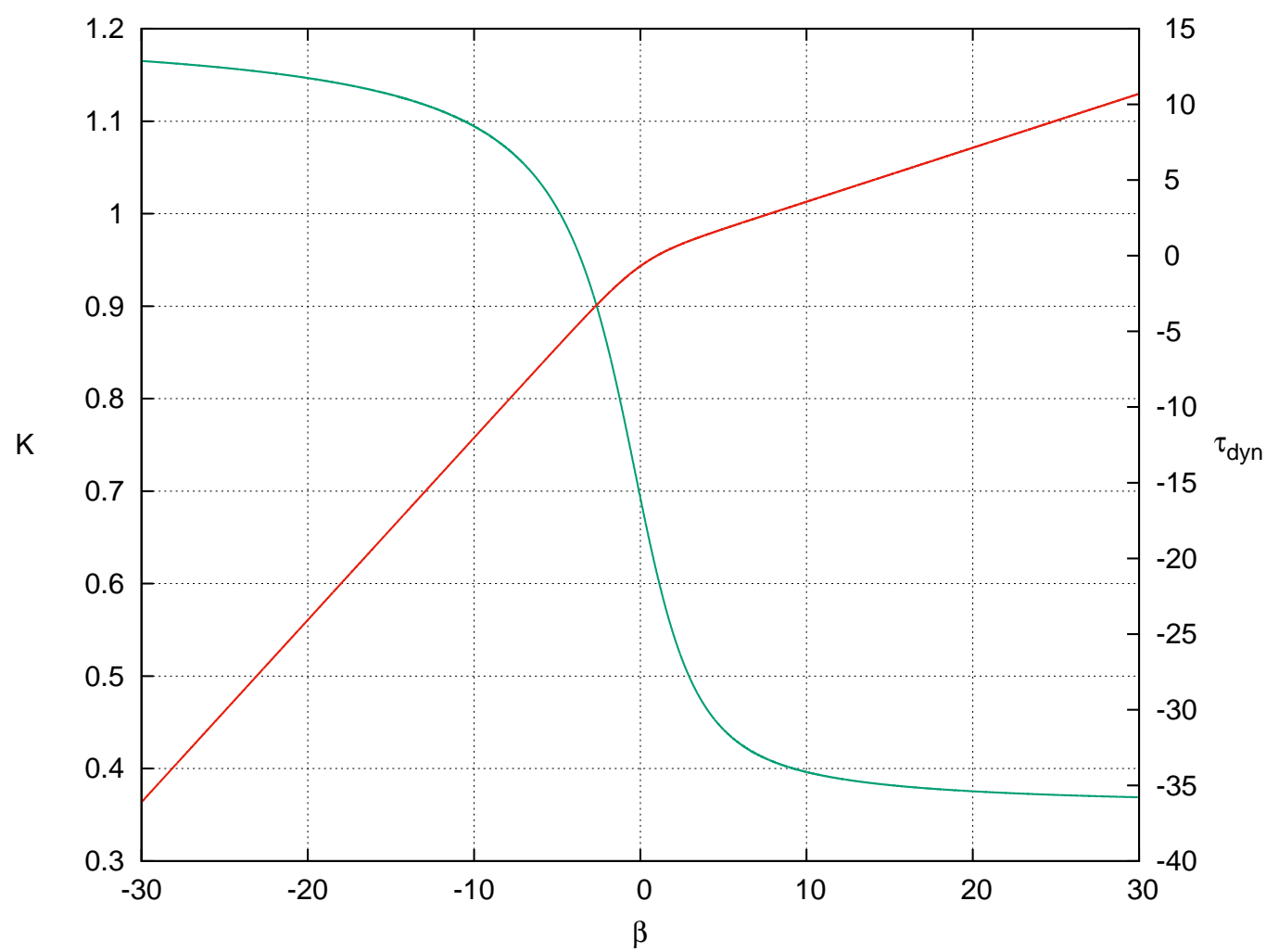

FIG. 1: (Color online) Rényi entropy $K(\beta)$ (green) and $\tau_{d y n}(\beta)$ (red) of the asymmetric triangular map as a function of $\beta$ for the parameter value $w=0.3$.

that is, $\tau_{d y n}(\beta)$ is linear if the absolute value of $\beta$ is large. The first derivative of $\tau_{d y n}$

$$
\gamma=\frac{w^{\beta} \ln w+(1-w)^{\beta} \ln (1-w)}{w^{\beta}+(1-w)^{\beta}}
$$

is plotted on Fig. 2. The minimum and maximum scaling indices are

$$
\gamma_{\min }=\lim _{\beta \rightarrow-\infty} \gamma=\ln w
$$

and

$$
\gamma_{\max }=\lim _{\beta \rightarrow+\infty} \gamma=\ln (1-w)
$$

if $w<1-w$.

The Fisher information expressed with the second derivative of $\tau_{d y n}$ (Eq. (39))

$$
\mathcal{F}_{\gamma}=\frac{w^{\beta}(1-w)^{\beta}}{\left[w^{\beta}+(1-w)^{\beta}\right]^{2}}[\ln w-\ln (1-w)]^{2}
$$






FIG. 2: (Color online) The scaling index $\gamma$ (green) and the Fisher information $\mathcal{F}_{\gamma}$ (red) (i. e. the first and second derivatives of $\tau_{d y n}(\beta)$ with respect to $\beta$ ) for the asymmetric triangular map as a function of $\beta$ for the parameter value $w=0.3$.

is plotted on Fig. 2. The Fisher information is a fundamental function of $\mathcal{F}_{g}$ given by Eq. (40) and is presented on Fig. 3. As the Fisher information is very small if the absolute value of $\beta$ is large

$$
\lim _{\beta \rightarrow-\infty} \mathcal{F}_{g}=\ln w
$$

and

$$
\lim _{\beta \rightarrow+\infty} \mathcal{F}_{g}=\ln (1-w),
$$

that is, $\mathcal{F}_{g}$ tends to the scaling index $\gamma$ in this case. The Legendre transform $\mathcal{F}_{\phi}$ that corresponds to the free energy of the standard thermodynamics is given by Eq. (42) and is shown on Fig. 3. As $\mathcal{F}_{\phi}=-\gamma / \beta$, we can see that $\mathcal{F}_{\phi} \rightarrow 0$ if $\beta \rightarrow \pm \infty$, while $\mathcal{F}_{\phi} \rightarrow \infty$ if $\beta \rightarrow-0$ and $\mathcal{F}_{\phi} \rightarrow-\infty$ if $\beta \rightarrow+0$.

Traditionally, multifractals are characterized by the functions $K(\beta)$ and $\tau_{d y n}(\beta)$. Here we propose to describe these objects by the Fisher information $\mathcal{F}_{\gamma}$ and its Legendre transforms. 


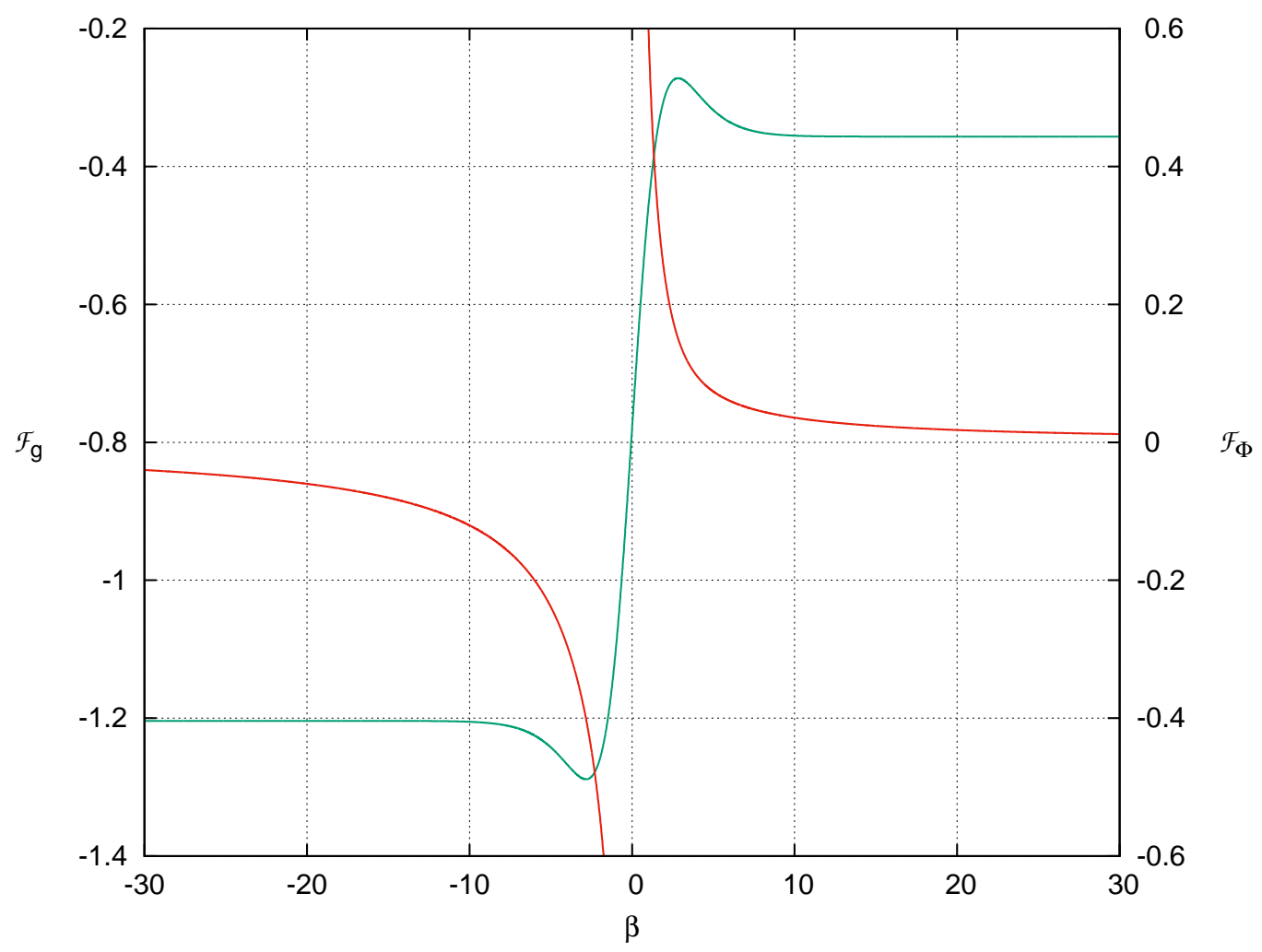

FIG. 3: (Color online) Legendre transforms $\mathcal{F}_{g}$ (green) and $\mathcal{F}_{\phi}$ (red) of the Fisher information $\mathcal{F}_{\gamma}$ for the asymmetric triangular map as a function of $\beta$ for the parameter value $w=0.3$.

They contain more details of the multifractal behaviour. As we can see on Figs. 2 and 3, the Fisher information $\mathcal{F}_{\gamma}$ and its Legendre transforms $\mathcal{F}_{g}$ and $\mathcal{F}_{\phi}$ reflect the change in $\tau_{d y n}$ in the vicinity of $\beta=0$ in different ways, providing different aspects of the fluctuations.

The specific heat $C_{d y n}$ ( Eq. (45)), which is closely related to the Fisher information $\mathcal{F}_{\gamma}$, is plotted on Fig. 4. The Fisher heat capacity (Eq. (46)) takes the form

$$
\mathcal{C}_{F}^{d y n}=\beta^{2} \frac{[\ln w-\ln (1-w)]^{3}}{\left[w^{\beta}+(1-w)^{\beta}\right]^{3}}\left[w^{\beta}-(1-w)^{\beta}\right] .
$$

$\mathcal{C}_{F}^{d y n}$ is presented on Fig. 4. We can see even in the traditional thermodynamic formalism (Fig. 1) that the most interesting behaviour is around $\beta=0$. The present Fisher formalism gives more detailed information on the characteristics in this region (Figs. 2 and 3). The Fisher information $\mathcal{F}_{\gamma}$ and the Fisher heat capacity describes the fluctuation in $\gamma$, they are sensitive to changes of higher order than its analogue of the usual formalism.

It is worth mentioning that the Rényi entropies $K(\beta)$ and the symbol-sequence probabilities of quantum-mechanical measurement process coincide with those of the present chaotic 


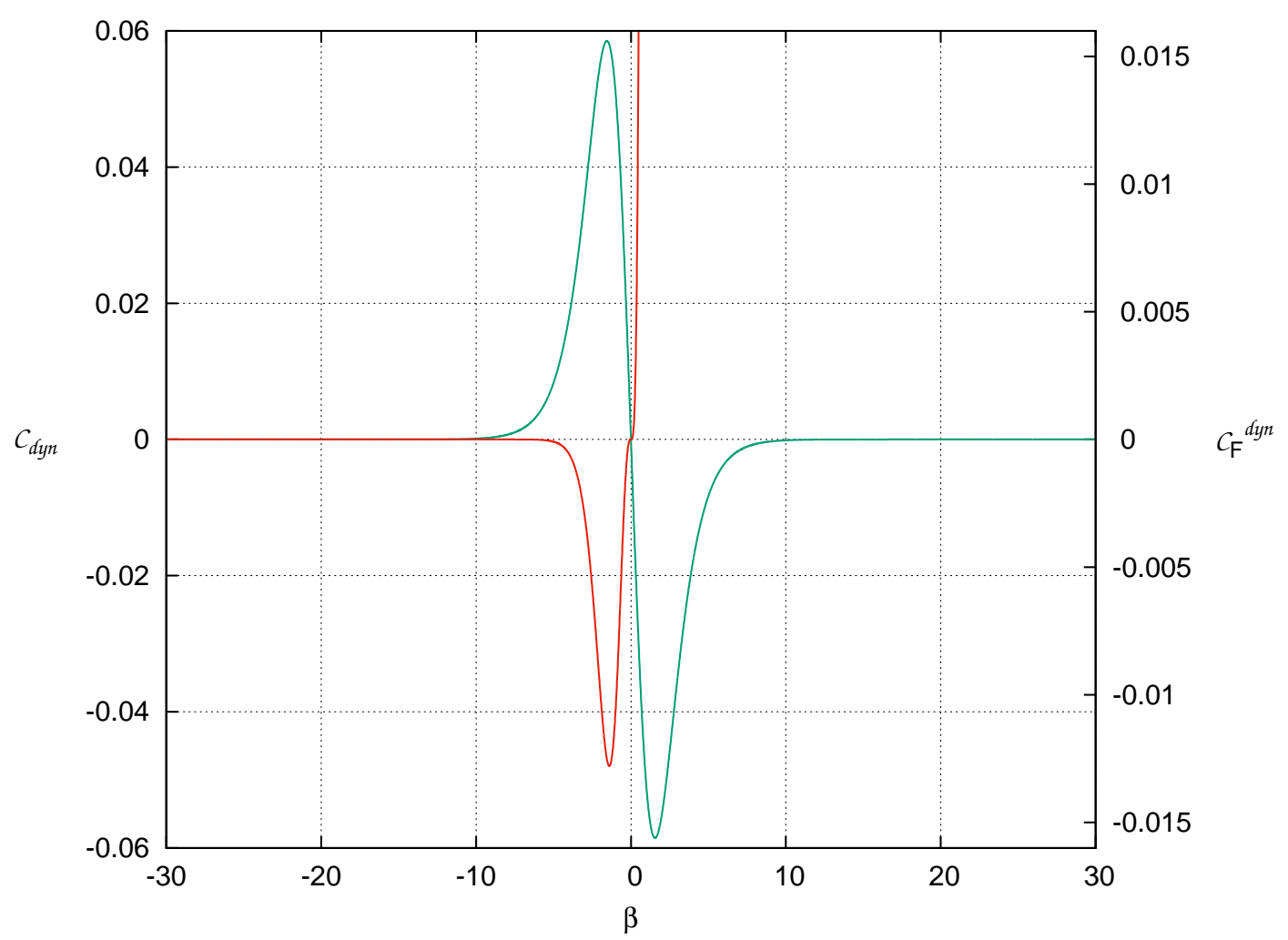

FIG. 4: (Color online) The heat capacity $C_{d y n}(\beta)$ (green) and the Fisher heat capacity $\mathcal{C}_{F}^{d y n}$ (red) for the asymmetric triangular map as a function of $\beta$ for the parameter value $w=0.3$.

map [8, 16-18]. The role of Fisher information and its Legendre transforms in quantummechanical measurement would be an interesting subject of further research.

In summary, the thermodynamical formalism based on Fisher information is presented to describe dynamical systems. The Fisher information and its Legendre transforms are useful quantities to characterize the dynamics of multifractals.

[1] R. A. Fisher, Proc. Cambridge Philos. Soc. 22 (1925) 700.

[2] R. Frieden, Physics from Fisher Information. A unification; Cambridge University Press, Cambridge, 1998.

[3] R. Frieden and B. H. Soffer, Phys. Rev. E 60, 48 (1999).

[4] R. Frieden and B. H. Soffer, Phys. Rev. E 52, 2274 (1995).

[5] F. Pennini and A. Plastino, Phys. Rev. E 71, 047102 (2005). 
[6] A. Porporato, Phys. Rev. E 89, 042126 (2014).

[7] B. Godó and Á. Nagy, Chaos 26, 083102 (2016).

[8] C. Beck and F. Schlögl, Thernodynamics of chaotic systems: An introduction, Cambridge Univ. Press, Cambridge, 1993.

[9] T. Tél, Z. Naturforsch, 43a, 1154 (1988).

[10] T. Tél, in Directions in Chaos 3, ed. Hao Bai-Lin, World Scientific, Singapore (1990).

[11] M. Kohmoto, Phys. Rev. A 37, 1345 (1988).

[12] M. Khatua, D. Chakraborty and P. K. Chattaraj, In. J. Quant. Chem. 113, 1747 (2013).

[13] A. Rényi, in Proceedings of Fourth Berkeley Symp. on Mathematics, Statistics and Probability, vol.1. Univ. California Press, Berkeley, 1961, pp.547.

[14] C. E. Shannon, Bell Syst. Tech. J. 27, 623 (1948).

[15] T. C. Halsey, M. H. Jensen, L. P. Kadanoff, I. Procaccia and B. I. Shraiman, Phys. Rev. A 33, 1141 (1986).

[16] C. Beck and D. Graudenz, Phys. Rev. A 46, 6265 (1992).

[17] E. Romera and F. de los Santos, Phys. Rev. E 78, 013837 (2008).

[18] K. Wiesner and J. P. Crutchfield, Nat. Comput. 9, 317 (2010). 
TABLE I: Thermodynamic variables and their analogues in chaotic dynamics in the usual thermodynamic and the Fisher information representation

\begin{tabular}{cccc}
\hline & Thermodynamics & \multicolumn{2}{c}{ Chaotic dynamics } \\
Entropy & Fisher information & Entropy & Fisher information \\
\hline$S$ & $\mathcal{F}_{S}$ & $g$ & $\mathcal{F}_{g}$ \\
$U$ & $\mathcal{F}_{U}$ & $\gamma$ & $\mathcal{F}_{\gamma}$ \\
$\Phi=S-\beta U$ & $U=\mathcal{F}_{S}-\beta \mathcal{F}_{U}$ & $-\tau(\beta)=g(\gamma)-\beta \gamma$ & $\gamma=\mathcal{F}_{g}-\beta \mathcal{F}_{\gamma}$ \\
$F=-T \Phi$ & $\mathcal{F}_{F}=-T U$ & $F=T \tau(\beta)$ & $\mathcal{F}_{\phi}=-T \gamma$ \\
$=U-T S$ & $=\mathcal{F}_{U}-T \mathcal{F}_{S}$ & $=\gamma-T g$ & $\mathcal{F}_{\gamma}-T \mathcal{F}_{g}$ \\
$S(U)$ & $\mathcal{F}_{S}\left(\mathcal{F}_{U}\right)$ & $g(\gamma)$ & $\mathcal{F}_{g}\left(\mathcal{F}_{\gamma}\right)$ \\
$U(S)$ & $\mathcal{F}_{U}\left(\mathcal{F}_{S}\right)$ & $\gamma(g)$ & $\mathcal{F}_{\gamma}\left(\mathcal{F}_{g}\right)$ \\
$S=\beta U+\Phi$ & $\mathcal{F}_{S}=\beta \mathcal{F}_{U}+U$ & $\beta=\beta \mathcal{F}_{\gamma}+\gamma$ \\
$U=T S+F$ & $\mathcal{F}_{U}=T \mathcal{F}_{S}+\mathcal{F}_{U}$ & $\gamma=T g+F$ & $\mathcal{F}_{\gamma}=T \mathcal{F}_{g}+\mathcal{F}_{\phi}$ \\
$\frac{\partial S}{\partial U}=\beta$ & $\frac{\partial \mathcal{F}_{S}}{\partial \mathcal{F}_{U}}=\beta$ & $\frac{\partial g}{\partial \gamma}=\beta$ & $\frac{\partial \mathcal{F}_{g}}{\partial \mathcal{F}_{\gamma}}=\beta$ \\
$\frac{\partial U}{\partial S}=T$ & $\frac{\partial \mathcal{F}_{U}}{\partial \mathcal{F}_{S}}=T$ & $\frac{\partial \gamma}{\partial g}=T$ & $\frac{\partial \mathcal{F}_{\gamma}}{\partial \mathcal{F}_{g}}=T$ \\
$\frac{\partial \Phi}{\partial \beta}=-U$ & $\frac{\partial U}{\partial \beta}=-\mathcal{F}_{U}$ & $-\frac{\partial \tau}{\partial \beta}=-\gamma$ & $\frac{\partial \gamma}{\partial \beta}=-\mathcal{F}_{\gamma}$ \\
$\frac{\partial F}{\partial T}=-S$ & $\frac{\partial \mathcal{F}_{F}}{\partial T}=-\mathcal{F}_{S}$ & $\frac{\partial F}{\partial T}=-S$ & $\frac{\partial \mathcal{F}_{\phi}}{\partial T}=-\mathcal{F}_{g}$ \\
\hline \hline & & & \\
\hline
\end{tabular}

